\title{
Inverse Median Location Problems with Variable Coordinates
}

\author{
Fahimeh Baroughi Bonab*† Rainer E. Burkard* \\ Behrooz Alizadeh ${ }^{* \dagger}$
}

April 2009

\begin{abstract}
Given $n+1$ points in $\mathbb{R}^{d}$ with nonnegative weights, the inverse 1 -median problem with variable coordinates consists in changing the coordinates of the given points at minimum cost such that a prespecified point in $\mathbb{R}^{d}$ becomes the 1 -median. The cost is proportional to the increase or decrease of the corresponding point coordinate. In case that the distances between points are measured by the rectilinear norm, it is shown that the inverse 1-median problem is $\mathcal{N} \mathcal{P}$-hard, but it can be solved by a pseudo-polynomial algorithm. If the point weights are assumed to be equal, the corresponding inverse problem can be reduced to $d$ continuous knapsack problems and is therefore solvable in $O(n d)$ time. In case that the squared Euclidean norm is used, we derive another efficient combinatorial algorithm which solves the problem in $O(n d)$ time. It is also shown that the inverse 1-median problem endowed with the Chebyshev norm in the plane is $\mathcal{N} \mathcal{P}$-hard. Another pseudo-polynomial algorithm is developed for this case.
\end{abstract}

Keywords: Location problem, 1-median, inverse optimization

\section{Introduction}

Inverse optimization problems have recently found a considerable interest. In an inverse optimization problem the task is to change the given parameters of the problem (like traffic connections or client rankings) at minimum cost so that a prespecified

\footnotetext{
${ }^{*}$ Graz University of Technology, Institute of Optimization and Discrete Mathematics, Steyrergasse 30, Graz, Austria. \{baroughi, burkard, alizadeh\}@opt.math.tugraz . at

${ }^{\dagger}$ Sahand University of Technology, Faculty of Basic Sciences for Engineering, Department of Applied Mathematics, Tabriz, Iran.

The third author acknowledges financial support by the NAWI-Project. This research has also been supported by the Austrian Science Fund (FWF) Project P18918-N18.
} 
solution becomes optimal. In 1992, Burton and Toint [7] investigated the inverse shortest path problem with an interesting application to geological sciences. Given a network, they change the edge lengths as little as possible so that a given path becomes the shortest path. Cai, Yang and Zhang [8] proved that the inverse 1-center location problem with variable edge lengths on general unweighted directed graphs is $\mathcal{N} \mathcal{P}$-hard, while the underlying center location problem is solvable in polynomial time. Burkard, Pleschiutschnig and Zhang [4,5] investigated the inverse 1-median problem with variable vertex weights and proved that the problem is solvable by a greedy-type algorithm in $O(n \log n)$ time if the underlying network is a tree or the location problem is defined in the plane (where distances are measured by the rectilinear or the Chebyshev norm) and in $O\left(n^{2}\right)$ time on cycles. On the other hand, Gassner [9] suggested an $O(n \log n)$ time algorithm for the inverse 1-maxian problem on a tree with variable edge lengths. In 2008, Burkard, Galavii and Gassner [3] solved the inverse Fermat-Weber problem with variable vertex weights in $O(n \log n)$ time for unit cost under the assumption that the prespecified point that should become a 1-median does not coincide with a given point in the plane. Yang and Zhang [14] considered the inverse vertex center problem with variable edge lengths on tree networks. They proposed an $O\left(n^{2} \log n\right)$ time algorithm for this problem where it is assumed that all the modified edge lengths remain positive. Alizadeh and Burkard [1] investigated the inverse absolute 1-center location problem on tree networks with variable edge lengths. They showed that the problem can be solved in $O\left(n^{2}\right)$ time by an exact algorithm provided that the topology of the tree does not change. Dropping this condition, they proposed an $O\left(n^{2} r\right)$ time exact algorithm for the general case where $r$ is the compressed depth of the underlying tree. Recently Alizadeh, Burkard and Pferschy [2] used a set of suitably extended AVL-search trees and developed a combinatorial algorithm which solves the inverse 1-center location problem with edge length augmentation in $O(n \log n)$ time. For a survey of inverse optimization the reader is referred to Heuberger [11].

In this paper we investigate the inverse 1-median problem with variable coordinates endowed with the rectilinear, the squared Euclidean or the Chebyshev norm. The paper is organized as follows: In the next section we introduce the inverse 1median problem with variable coordinates in $\mathbb{R}^{d}$ and formulate the problem as a nonlinear optimization model. In Section 3 we show that this problem is $\mathcal{N} \mathcal{P}$-hard if the rectilinear norm is used, but it can be solved by a pseudo-polynomial algorithm. Next we consider equal weights and show that in this case the problem is polynomially solvable. In Section 4 we investigate the inverse 1-median problem in $\mathbb{R}^{d}$ endowed with the squared Euclidean norm and show that this problem can be solved efficiently in polynomial time. Finally, in Section 5 we consider the inverse 1-median problem in the plane endowed with the Chebyshev norm and show that this problem is $\mathcal{N} \mathcal{P}$-hard. In this case the given bounds on the distances between new and old points prevent require a new solution strategy. It is shown that this problem can be solved in pseudo-polynomial time. 


\section{The 1-median problem and the inverse 1-median prob- lem with variable coordinates}

Let $P_{i}=\left(x_{i 1}, x_{i 2}, \ldots, x_{i d}\right), i=1, \ldots, n$, be $n$ distinct points (called customers) which are given in the $d$-dimensional real space $\mathbb{R}^{d}$. Let $w_{i}, i=1, \ldots, n$, be nonnegative weights assigned to the customers. In the classical 1-median problem the goal is to find a new point $P_{0} \in \mathbb{R}^{d}$ (called supplier), which minimizes

$$
f(P)=\sum_{i=1}^{n} w_{i} d\left(P_{i}, P\right) \quad \text { for all } P=\left(y_{1}, \ldots, y_{d}\right) \in \mathbb{R}^{d},
$$

where $d\left(P_{i}, P\right)$ is the distance between points $P_{i}$ and $P$. In this case $P_{0}$ is called a 1-median of the points $P_{i}$.

Now we are going to state the inverse 1-median problem with variable coordinates in $\mathbb{R}^{d}$ : Let $n+1$ distinct points $P_{i}=\left(x_{i 1}, x_{i 2}, \ldots, x_{i d}\right), i=0,1, \ldots, n$, with nonnegative weights $w_{i}$ be given. We want to modify the coordinates of the points at minimum cost such that $P_{0}$ becomes a 1-median. Assume that we incur the nonnegative cost $c_{i j}^{+}$and $c_{i j}^{-}, 1 \leq j \leq d$, if the coordinate $x_{i j}$ is increased and decreased by one unit, respectively. Let $q_{i j}^{+}$and $q_{i j}^{-}$be the amounts by which the coordinate $x_{i j}$ is increased and decreased. Moreover, we suppose that $q_{i j}^{+}$and $q_{i j}^{-}$obey the upper bounds $u_{i j}^{+}$ and $u_{i j}^{-}$, respectively. For convenience we set $u_{i}^{+}=\left(u_{i 1}^{+}, \ldots, u_{i d}^{+}\right), u_{i}^{-}=\left(u_{i 1}^{-}, \ldots, u_{i d}^{-}\right)$, $q_{i}^{+}=\left(q_{i 1}^{+}, \ldots, q_{i d}^{+}\right)$and $q_{i}^{-}=\left(q_{i 1}^{-}, \ldots, q_{i d}^{-}\right)$. Thus the inverse 1-median problem with variable coordinates in $\mathbb{R}^{d}$ can be formulated as the following nonlinear optimization model:

(P) minimize $\sum_{i=1}^{n} \sum_{j=1}^{n}\left(c_{i j}^{+} q_{i j}^{+}+c_{i j}^{-} q_{i j}^{-}\right)$

$$
\begin{aligned}
& \text { subject to } \sum_{i=1}^{n} w_{i} d\left(P_{i}^{*}, P_{0}\right) \leq \sum_{i=1}^{n} w_{i} d\left(P_{i}^{*}, P\right)+w_{0} d\left(P_{0}, P\right) \text { for all } P \in \mathbb{R}^{d} \text {, } \\
& P_{i}^{*}=P_{i}+q_{i}^{+}-q_{i}^{-} \quad \text { for } i=1, \ldots, n, \\
& 0 \leq q_{i}^{+} \leq u_{i}^{+} \quad \text { for } i=1, \ldots, n \text {, } \\
& 0 \leq q_{i}^{-} \leq u_{i}^{-} \quad \text { for } i=1, \ldots, n \text {. }
\end{aligned}
$$

In the case that $d$ is the rectilinear distance or the squared Euclidean distance in $\mathbb{R}^{d}$, inequality (2) is separable. This allows to split the problem into $d$ inverse 1-median problems on a line. In case of the squared Euclidean distance the function $f$ defined by (1) is convex and differentiable in $y_{1}, \ldots, y_{d}$. Therefore by computing the partial derivatives and finding their roots we get the unique minimum of $f$.

Observation 2.1 In case of the rectilinear or the squared Euclidean distance the given point $P_{0}$ is a 1-median of the points $P_{i}, i=0,1, \ldots, n$, if and only if for every $j=1, \ldots, d$, point $x_{0 j}$ is a 1-median of points $x_{i j}, i=0, \ldots, n$, on the line. 
Observation 2.1 implies that constraint $(2)$ of problem $(\mathrm{P})$ is equivalent with the constraints

$$
\sum_{i=1}^{n} w_{i} d\left(x_{i j}^{*}, x_{0 j}\right) \leq \sum_{i=1}^{n} w_{i} d\left(x_{i j}^{*}, y\right)+w_{0} d\left(x_{0 j}, y\right) \text { for all } y \in \mathbb{R}, \quad \text { for } j=1, \ldots, d \text {. }
$$

Thus the solution of the inverse problem $(\mathrm{P})$ can be obtained through the solutions of the following $d$ nonlinear optimization problems $\left(\mathrm{P}_{\mathrm{j}}\right), j=1, \ldots, d$,

$$
\begin{array}{rll}
\left(\mathrm{P}_{\mathrm{j}}\right) \text { minimize } & \sum_{i=1}^{n}\left(c_{i j}^{+} q_{i j}^{+}+c_{i j}^{-} q_{i j}^{-}\right) & \\
\text {subject to } & \sum_{i=1}^{n} w_{i} d\left(x_{i j}^{*}, x_{0 j}\right) \leq \sum_{i=1}^{n} w_{i} d\left(x_{i j}^{*}, y\right)+w_{0} d\left(x_{0 j}, y\right) \quad \text { for all } y \in \mathbb{R}, \\
& x_{i j}^{*}=x_{i j}+q_{i j}^{+}-q_{i j}^{-} & \text {for } i=1, \ldots, n, \\
& 0 \leq q_{i j}^{+} \leq u_{i j}^{+} & \text {for } i=1, \ldots, n, \\
& 0 \leq q_{i j}^{-} \leq u_{i j}^{-} & \text {for } i=1, \ldots, n .
\end{array}
$$

Therefore we get the following lemma:

Lemma 2.2 An optimal solution of the inverse 1-median problem with variable coordinates in $\mathbb{R}^{d}$ under the rectilinear or the squared Euclidean norm can be obtained by solving d inverse 1-median problems on a line.

In the next two sections by recalling basic properties of the classical 1-median location problems we develop exact solution algorithms for the inverse 1-median problem on a line endwoed with the rectilinear (or the squared Euclidean) norm which consequently lead to the solution of the problems under investigation in $\mathbb{R}^{d}$ according to Lemma 2.2 .

\section{The inverse 1-median problem under the rectilinear norm}

In this section first we investigate the inverse 1-median problem with variable coordinates on a line endowed with the rectilinear norm and show that this problem is $\mathcal{N} \mathcal{P}$-hard. Since it is equivalent to a binary knapsack problem, it can be solved by a pseudo-polynomial algorithm. Then by using the solution method for the inverse 1 -median problem on the line, we solve the inverse 1 -median problem in $\mathbb{R}^{d}$. Next we consider the inverse 1 -median problem in $\mathbb{R}^{d}$ with equal weights and show that this problem is polynomialy solvable.

\subsection{The inverse 1-median problem with arbitrary weights}

Assume that $X=\left\{x_{0}, x_{1}, \ldots, x_{n}\right\}$ is a set of $n+1$ points on a line. The cost for increasing and decreasing the coordinate $x_{i}$ is denoted by $c_{i}^{+}$and $c_{i}^{-}$, respectively. 
Moreover, Let $*$ be any of the relations $<, \leq,>, \geq$. In the following we use the notations

$$
\begin{gathered}
I_{*}(X)=\left\{i: x_{i} * x_{0}, 0 \leq i \leq n\right\}, \\
X_{*}=\left\{x_{i} \in X: i \in I_{*}(X)\right\}, \\
W_{*}(X)=\sum_{i \in I_{*}(X)} w_{i} .
\end{gathered}
$$

An optimality criterion for the classical 1-median problem on a line is:

Lemma 3.1 (Optimality criterion) [10]

The point $x_{0}$ is a 1-median of $x_{i}, i=0, \ldots, n$, on the line if and only if

$$
W_{<}(X)-W_{\geq}(X) \leq 0 \quad \text { and } \quad W_{>}(X)-W_{\leq}(X) \leq 0 .
$$

In order to simplify the notations, we use $I_{*}$ and $W_{*}$ instead of $I_{*}(X)$ and $W_{*}(X)$, respectively, in this section.

Let us assume that $x_{0}$ is not the median of the points $x_{i}, i=0, \ldots, n$, on the line. We modify the coordinates of $x_{i}$ in order to achieve that $x_{0}$ becomes a 1-median.

If

$$
W_{<}-W_{\geq}>0,
$$

then any coordinate change for points in $X_{\geq}$does not help to restore (3). Therefore, we have to move some points of $X_{<}$to the set $X_{\geq}$under minimum total cost until the optimality criterion is satisfied. Obviously, some points of $X_{<}$have to coincide with $x_{0}$. The analogous situation applies for

$$
W_{>}-W_{\leq}>0 .
$$

Thus for solving the inverse 1-median problem on the line we get

Lemma 3.2 i) If inequality (4) holds, then it is sufficient that some points of the set $X_{<}$are moved to $x_{0}$ by increasing their coordinates at minimum cost, such that $x_{0}$ becomes a 1-median.

ii) If inequality (5) holds, then it is sufficient that some points of the set $X_{>}$are moved to $x_{0}$ by decreasing their coordinates at minimum cost, such that $x_{0}$ becomes a 1-median.

Both cases can be formulated as a knapsack problem. Let $W=\sum_{i=0}^{n} w_{i}$ be the sum of all point weights. In case of inequality (4) we define

$$
L=\left\{i: i \in I_{<}, u_{i}^{+} \geq x_{0}-x_{i}\right\}
$$

and

$$
W L=\sum_{i \in L} w_{i}
$$

If

$$
W L<W_{<}-\frac{W}{2}
$$


then any coordinate increase for points in $X_{<}$does not help to restore (3). Thus the problem has no solution. Otherwise, we introduce the binary variable $s_{i}$, where $s_{i}=1$ means that point $x_{i}$ is moved to coincide with $x_{0}$. Now we consider the following knapsack problem which is equivalent with the considered inverse 1-median problem on a line.

$$
\begin{array}{ll}
\left(\mathrm{KP}_{1}\right) \text { minimize } & \sum_{i \in L} c_{i}^{+}\left(x_{0}-x_{i}\right) s_{i} \\
\text { subject to } & \sum_{i \in L} w_{i} s_{i} \geq \frac{1}{2}\left(W_{<}-W_{\geq}\right), \\
& s_{i} \in\{0,1\} \quad \text { for all } i \in L .
\end{array}
$$

$\left(\mathrm{KP}_{1}\right)$ can be rewritten as the following knapsack problem:

$$
\begin{array}{ll}
\left(\mathrm{KP}_{2}\right) \quad \text { maximize } & \sum_{i \in L} c_{i}^{+}\left(x_{0}-x_{i}\right) s_{i}^{\prime} \\
\text { subject to } & \sum_{i \in L} w_{i} s_{i}^{\prime} \leq \sum_{i \in L} w_{i}-\frac{1}{2}\left(W_{<}-W_{\geq}\right), \\
& s_{i}^{\prime} \in\{0,1\} \quad \text { for all } i \in L .
\end{array}
$$

Problem $\left(\mathrm{KP}_{2}\right)$ is $\mathcal{N} \mathcal{P}$-hard, but it can be solved by a pseudo-polynomial algorithm in $O(n W)$ time, see e.g., $[12,13]$.

The case of inequality (5) can be treated in an analogous way. Since every knapsack problem instance describes also an instance of the inverse 1-median problem on a line with variable coordinates, we get

Proposition 3.3 The inverse 1-median problem with variable coordinates with $(n+$ 1) points on a line is $\mathcal{N} \mathcal{P}$-hard, but it can be solved by a pseudo-polynomial algorithm with time complexity $O(n W)$, where $W$ is the sum of all weights.

For solving the inverse 1-median problem in $\mathbb{R}^{d}$ endowed with the rectilinear norm we shall apply now the following Algorithm 1.

Theorem 3.4 The inverse 1-median problem with variable coordinates with $(n+1)$ points in $\mathbb{R}^{d}, d \geq 1$, under the rectilinear norm is $\mathcal{N} \mathcal{P}$-hard, but it can be solved by a pseudo-polynomial algorithm with complexity of $O(d n W)$, where $W$ is the sum of all weights.

\subsection{The inverse 1-median problem with equal weights}

If in the problem under investigation it is assumed that all the weights are equal, i.e.,

$$
w_{i}=\tilde{w} \quad \text { for } i=0, \ldots, n,
$$




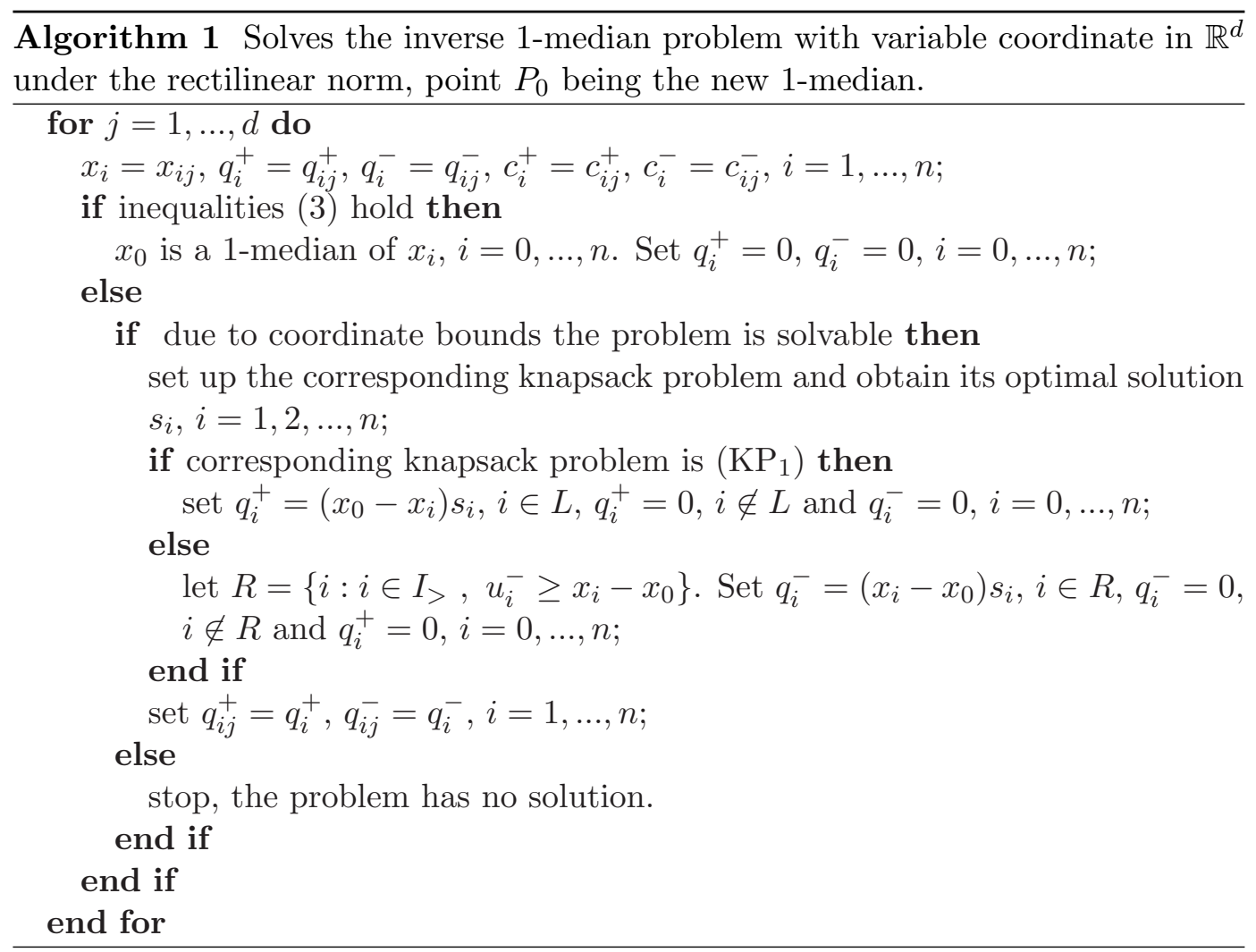

then problem $\left(\mathrm{KP}_{1}\right)$ becomes the following special knapsack problem:

$$
\begin{aligned}
\left(\mathrm{KP}_{3}\right) \quad \text { minimize } & \sum_{i \in L} c_{i}^{+}\left(x_{0}-x_{i}\right) s_{i} \\
\text { subject to } & \sum_{i \in L} s_{i} \geq \frac{1}{2}\left(\left|X_{<}\right|-\left|X_{\geq}\right|\right), \\
& s_{i} \in\{0,1\} \quad \text { for all } i \in L .
\end{aligned}
$$
for

Let $\varphi$ be a permutation of the set $L=\{1, \ldots, t\}$ such that $\tilde{c}_{\varphi(1)} \leq \ldots \leq \tilde{c}_{\varphi(t)}$ holds

$$
\tilde{c}_{\varphi(i)}=c_{\varphi(i)}^{+}\left(x_{0}-x_{\varphi(i)}\right) \quad \text { for all } i \in L .
$$

Moreover, let

$$
c=\frac{1}{2}\left(\left|X_{<}\right|-\left|X_{\geq}\right|\right) .
$$

Definition 3.5 An item $\varphi(r) \in\{\varphi(1), \ldots, \varphi(t)\}$ is called the critical item of problem $\left(\mathrm{KP}_{3}\right)$, if

$$
\sum_{i=1}^{r-1} w_{i}=\tilde{w}(r-1) \leq c \quad \text { and } \quad \sum_{i=1}^{r} w_{i}=\tilde{w} r>c .
$$


The following proposition shows how an optimal solution of the problem $\left(\mathrm{KP}_{3}\right)$ can be found if we know the critical item.

Proposition 3.6 The optimal solution $s^{*}$ of problem $\left(\mathrm{KP}_{3}\right)$ is

$$
\begin{aligned}
& s_{\varphi(i)}^{*}=1 \quad \text { for } i=1, \ldots, r-1, \\
& s_{\varphi(r)}^{*}= \begin{cases}0 & \tilde{w}(r-1)=c, \\
1 & \text { otherwise, }\end{cases} \\
& s_{\varphi(i)}^{*}=0 \quad \text { for } i=r+1, \ldots, t .
\end{aligned}
$$

Based on the definition of a critical item for problem $\left(\mathrm{KP}_{3}\right)$, and on a critical item of the continuous knapsack problem, see i.e., [12], we get

Observation 3.7 Problem $\left(\mathrm{KP}_{3}\right)$ and its corresponding continuous model have the same critical item.

An analogous argument can be applied in case of inequality (5). Since the critical item of a continuous knapsack problem can be found in linear time [6], we conclude the following theorem:

Theorem 3.8 The inverse 1-median problem with variable coordinates with $(n+1)$ points in $\mathbb{R}^{d}, d \geq 1$ under the rectilinear norm and equal weights can be solved in $O(d n)$ time.

\section{The inverse 1-median problem under the squared Eu- clidean norm}

In this section we investigate the inverse 1-median problem with variable coordinates in $\mathbb{R}^{d}$ where the distances are measured by the squared Euclidean norm. We develop an exact solution method for the problem which runs in $O(d n)$ time.

First let us introduce the following optimality criterion for the classical 1-median problem on a line.

Lemma 4.1 (Optimality criterion) [10]

The point $x_{0}$ is called a 1-median of $x_{i}, i=0, \ldots, n$, on a line endowed with the squared Euclidean norm if and only if

$$
x_{0}=\frac{\sum_{i=0}^{n} w_{i} x_{i}}{\sum_{i=0}^{n} w_{i}} .
$$

Now assume that $x_{0}$ is not the median of points $x_{i}, i=0, \ldots, n$, on the line. We modify the coordinates of $x_{i}$ in order to achieve that $x_{0}$ becomes a 1 -median.

If

$$
x_{0}>\frac{\sum_{i=0}^{n} w_{i} x_{i}}{\sum_{i=0}^{n} w_{i}},
$$

then by decreasing the coordinates of points $x_{i}, i=1, \ldots, n$, the optimality criterion can never be fulfilled. Therefore, we have to increase the coordinates of some points 
$x_{i}, i=1, \ldots, n$, at minimum total cost such that the optimality criterion is satisfied. The analogous situation applies for

$$
x_{0}<\frac{\sum_{i=0}^{n} w_{i} x_{i}}{\sum_{i=0}^{n} w_{i}}
$$

Thus we get the following lemma.

Lemma 4.2 The following two statements hold:

i) If the inequality (7) is satisfied, then it is sufficient to increase the coordinates of some $x_{i}, i=1, \ldots, n$, at minimum cost such that $x_{0}$ becomes a 1-median.

ii) If the inequality (8) is satisfied, then it is sufficient to decrease the coordinates of some $x_{i}, i=1, \ldots, n$, at minimum cost such that $x_{0}$ becomes a 1-median.

Both cases can be formulated as a continuous knapsack problem. In case of inequality (7), we have to increase the coordinates of some $x_{i}, i=1, \ldots, n$, by amounts $q_{i}^{+} \in \mathbb{R}^{+}$at minimum cost and with respect to the modification bounds such that

$$
\sum_{i=1}^{n} w_{i} q_{i}^{+}=\sum_{i=1}^{n} w_{i} x_{0}-\sum_{i=1}^{n} w_{i} x_{i}
$$

holds. Now let

$$
D=\sum_{i=1}^{n} w_{i} x_{0}-\sum_{i=1}^{n} w_{i} x_{i}
$$

If

$$
\sum_{i=1}^{n} w_{i} u_{i}^{+}<D
$$

then any increase for points $x_{i}, i=1, \ldots, n$, does not help to restore (6). Thus the problem under investigation has no solution. Otherwise, consider

$$
\begin{aligned}
& \text { (LP) minimize } \sum_{i=1}^{n} c_{i}^{+} q_{i}^{+} \\
& \text {subject to } \sum_{i=1}^{n} w_{i} q_{i}^{+}=D \text {, } \\
& 0 \leq q_{i}^{+} \leq u_{i}^{+} \quad \text { for } i=1, \ldots, n \text {. }
\end{aligned}
$$

By the transformations

$$
y_{i}=\frac{q_{i}^{+}}{u_{i}^{+}} \quad, \quad e_{i}=c_{i}^{+} u_{i}^{+} \quad, \quad \tilde{w}_{i}=w_{i} u_{i}^{+},
$$

problem (LP) can be transformed to the following continuous knapsack problem

$$
\begin{aligned}
\left(\mathrm{KP}_{4}\right) \quad \text { minimize } & \sum_{i=1}^{n} e_{i} y_{i} \\
\text { subject to } & \sum_{i=1}^{n} \tilde{w}_{i} y_{i}=D, \\
& 0 \leq y_{i} \leq 1 \quad \text { for } i=1, \ldots, n .
\end{aligned}
$$


which it can be solved in $O(n)$ time by the method mentioned in [12].

The case of inequality (8) can be treated in an analogous way. Thus we get

Proposition 4.3 The inverse 1-median problem with variable coordinates with $(n+$ 1) points on a line under the squared Euclidean norm can be solved in $O(n)$ time.

Now based on all concepts mentioned above we summarize our solution procedure for the inverse 1-median problem in $\mathbb{R}^{d}$ as Algorithm 2.

Algorithm 2 Solves the inverse 1-median problem with variable coordinates in $\mathbb{R}^{d}$ under the squared Euclidean norm, point $P_{0}$ being the new 1-median.

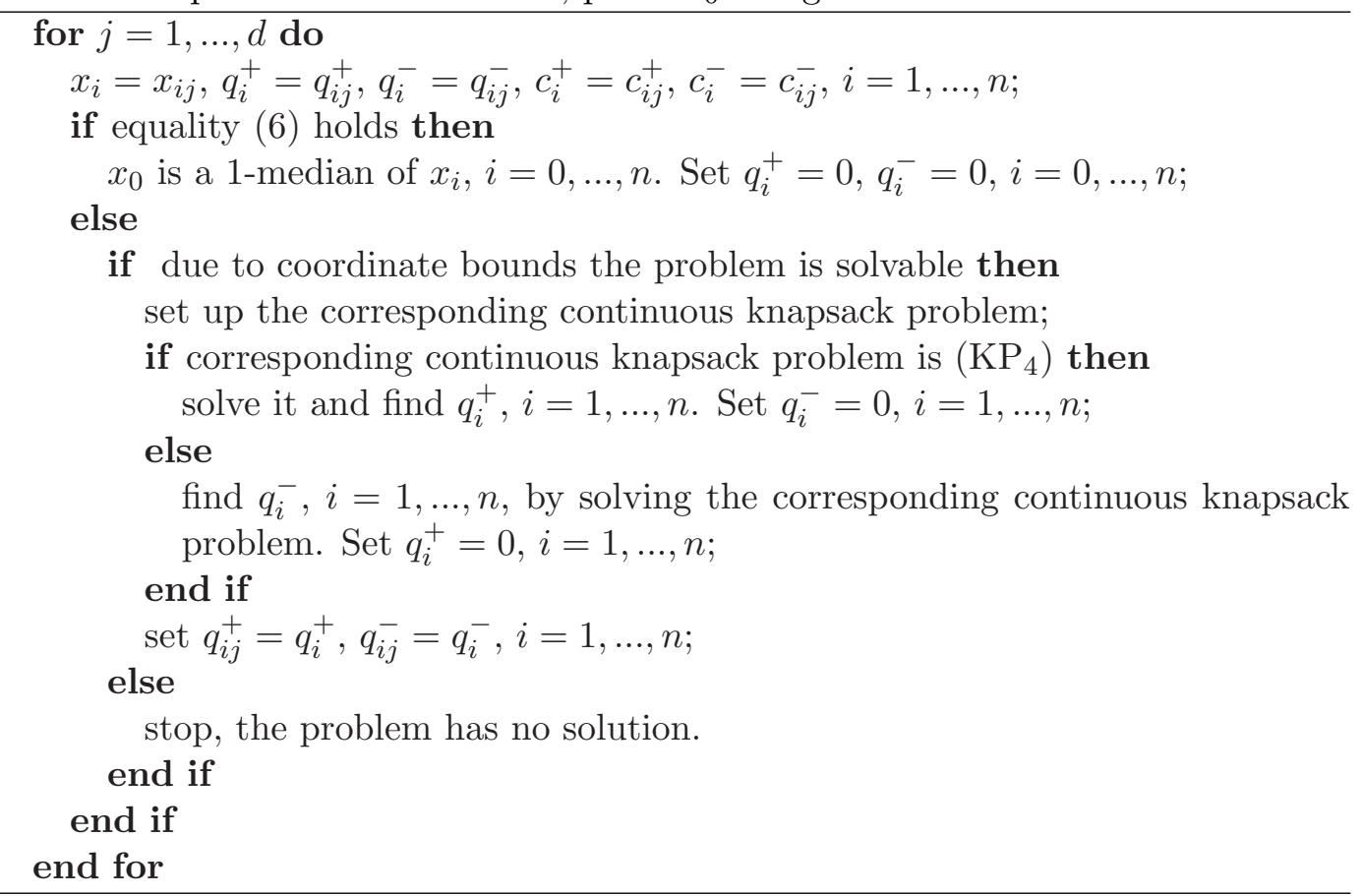

Theorem 4.4 The inverse 1-median problem with variable coordinates with $(n+1)$ points in $\mathbb{R}^{d}, d \geq 1$, under the squared Euclidean norm can be solved in $O(d n)$ time.

\section{The inverse 1-median problem under the Chebyshev norm}

In this section we investigate the inverse 1-median problem with variable coordinates in the plane endowed with the Chebyshev norm and show that the problem is $\mathcal{N} \mathcal{P}$ hard. We present a dynamic programming algorithm which solves the problem under investigation in $O\left(n W^{2}\right)$ time. Moreover, some special cases of the problem are investigated. It is shown that the inverse 1-median problem with unit weights is polynomially solvable. It is also shown that the unbounded inverse 1-median problem is $\mathcal{N} \mathcal{P}$-hard, but it can be solved by a pseudo-polynomial algorithm with time complexity $O(n W)$. 


\subsection{The general case}

The distance between the points $P_{i}=\left(x_{i}, y_{i}\right)$ and $P_{j}=\left(x_{j}, y_{j}\right)$ with respect to the rectilinear norm can be written as

$$
d_{1}\left(\left(x_{i}, y_{i}\right),\left(x_{j}, y_{j}\right)\right)=\left|x_{i}-x_{j}\right|+\left|y_{i}-y_{j}\right|
$$

and with respect to the Chebyshev norm as

$$
d_{\infty}\left(\left(x_{i}, y_{i}\right),\left(x_{j}, y_{j}\right)\right)=\max \left(\left|x_{i}-x_{j}\right|,\left|y_{i}-y_{j}\right|\right) .
$$

The following lemma shows a connection between the rectilinear and the Chebyshev norm in the plane.

Lemma 5.1 [10] For $P=(x, y) \in \mathbb{R}^{2}$, let $T(P)=\left(\frac{1}{2}(x+y), \frac{1}{2}(-x+y)\right)$. Then for all $P_{i}=\left(x_{i}, y_{i}\right)$ and $P_{j}=\left(x_{j}, y_{j}\right) \in \mathbb{R}^{2}$ :

$$
d_{\infty}\left(P_{i}, P_{j}\right)=d_{1}\left(T\left(P_{i}\right), T\left(P_{j}\right)\right)=d_{1}\left(\left(\tilde{x}_{i}, \tilde{y}_{i}\right),\left(\tilde{x_{j}}, \tilde{y}_{j}\right)\right)=d_{1}\left(\tilde{P}_{i}, \tilde{P}_{j}\right) .
$$

Now we introduce the inverse 1-median problem in the plane with respect to the Chebyshev norm. Let $n+1$ distinct points $P_{i}=\left(x_{i}, y_{i}\right), i=0,1, \ldots, n$, with nonnegative weights $w_{i}$ be given. We want to modify the coordinates of points at minimum cost such that $P_{0}$ becomes the 1-median. Suppose that we incur the nonnegative cost $c_{i}$, if the coordinate $x_{i}$ (or $y_{i}$ ) of $P_{i}$ is increased or decreased. We assume that the distance between points $P_{i}, i=1, \ldots, n$, and new points $P_{i}^{*}$, $i=1, \ldots, n$ are only allowed to be within certain bounds $u_{i}$. Thus the inverse 1 median problem can be expressed as follows:

Find new points $P_{i}^{*}, i=1, \ldots, n$, such that point $P_{0}$ is a 1 -median with respect to new points $P_{i}^{*}$, the distance between points $P_{i}^{*}$ and $P_{i}$ is bounded by $u_{i}$ for $i=1, \ldots, n$, and the total cost

$$
\sum_{i=1}^{n} c_{i} d_{\infty}\left(P_{i}^{*}, P_{i}\right)
$$

for changing the coordinates of points becomes minimum. Thus the inverse 1-median problem can be formulated as the following nonlinear optimization problem:

$$
\begin{aligned}
\left(\mathrm{P}_{1}\right) \text { minimize } & \sum_{i=1}^{n} c_{i} d_{\infty}\left(P_{i}^{*}, P_{i}\right) \\
\text { subject to } & \sum_{i=1}^{n} w_{i} d_{\infty}\left(P_{i}^{*}, P_{0}\right) \leq \sum_{i=1}^{n} w_{i} d_{\infty}\left(P_{i}^{*}, P\right)+w_{0} d_{\infty}\left(P_{0}, P\right) \text { for all } P \in \mathbb{R}^{2}, \\
& 0 \leq d_{\infty}\left(P_{i}^{*}, P_{i}\right) \leq u_{i} \quad \text { for } i=1, \ldots, n .
\end{aligned}
$$


Based on Lemma 5.1 problem $\left(\mathrm{P}_{1}\right)$ can be written as an inverse 1-median problem with respect to the rectilinear norm, i.e.,

$\left(\mathrm{P}_{2}\right) \quad$ minimize $\sum_{i=1}^{n} c_{i} d_{1}\left(\tilde{P}_{i}^{*}, \tilde{P}_{i}\right)$

subject to $\sum_{i=1}^{n} w_{i} d_{1}\left(\tilde{P}_{i}^{*}, \tilde{P}_{0}\right) \leq \sum_{i=1}^{n} w_{i} d_{1}\left(\tilde{P}_{i}^{*}, \tilde{P}\right)+w_{0} d_{1}\left(\tilde{P}_{0}, \tilde{P}\right)$ for all $\tilde{P} \in \mathbb{R}^{2}$,

$0 \leq d_{1}\left(\tilde{P}_{i}^{*}, \tilde{P}_{i}\right) \leq u_{i} \quad$ for $i=1, \ldots, n$.

Note that problem $\left(\mathrm{P}_{2}\right)$ and problem $(\mathrm{P})$ with $d=2$ are not the same. A main difference between these two problems is that in problem $\left(\mathrm{P}_{2}\right)$ the given bounds $u_{i}, i=1, \ldots, n$, may not allow to separate this problem into two inverse 1-median problems on lines. Hence, it is required that we develop a different method for problem $\left(\mathrm{P}_{2}\right)$ in general.

Assume that $\tilde{P}_{0}$ is not the median of points $\tilde{P}_{i}, i=0, \ldots, n$, in the plane. We modify the coordinates of $\tilde{P}_{i}$ in order to achieve that $\tilde{P}_{0}$ becomes a median.

Let us assume that $\tilde{P}_{i}^{*}=\left(\tilde{x}_{i}^{*}, \tilde{y}_{i}^{*}\right), i=1, \ldots, n$, are solutions of problem $\left(\mathrm{P}_{2}\right)$. Therefore

$$
P_{i}^{*}=\left(x_{i}^{*}, y_{i}^{*}\right)=T^{-1}\left(\tilde{P}_{i}^{*}\right)=\left(\tilde{x}_{i}^{*}-\tilde{y}_{i}^{*}, \tilde{x}_{i}^{*}+\tilde{y}_{i}^{*}\right) \text { for } i=1, \ldots, n,
$$

are solutions of the inverse 1-median problem endowed with the Chebyshev norm.

Assume that $X=\left\{\tilde{x}_{0}, \tilde{x}_{1}, \ldots, \tilde{x}_{n}\right\}$ and $Y=\left\{\tilde{y}_{0}, \tilde{y}_{1}, \ldots, \tilde{y}_{n}\right\}$. Consider $I_{*}(X)$, $I_{*}(Y), X_{*}, Y_{*}, W_{*}(X)$ and $W_{*}(Y)$ according to Section 3. As an immediate consequence of Observation 2.1 and Lemma 3.1 we get the following corollary:

Corollary 5.2 (Optimality criterion)

The point $\tilde{P}_{0}$ is a 1-median of $\tilde{P}_{i}, i=0, \ldots, n$, in the plane equipped with the rectilinear norm if and only if

$$
\begin{array}{ll}
W_{<}(X)-W_{\geq}(X) \leq 0 & , \quad W_{>}(X)-W_{\leq}(X) \leq 0, \\
W_{<}(Y)-W_{\geq}(Y) \leq 0 & , \quad W_{>}(Y)-W_{\leq}(Y) \leq 0 .
\end{array}
$$

Based on Corollary 5.2 and the concepts mentioned in Section 3 we conclude:

Lemma 5.3 For solving problem $\left(\mathrm{P}_{2}\right)$ it is sufficient that some points $\tilde{P}_{i}=\left(\tilde{x}_{i}, \tilde{y}_{i}\right)$, $i=1, \ldots, n$, are moved either to one of the points $\tilde{P}_{i}^{*}=\left(\tilde{x}_{i}, \tilde{y}_{0}\right)$, or $\tilde{P}_{i}^{*}=\left(\tilde{x}_{0}, \tilde{y}_{i}\right)$ or to $\tilde{P}_{i}^{*}=\left(\tilde{x}_{0}, \tilde{y}_{0}\right)$ by increasing or decreasing their coordinates at minimum cost subject to the modification bounds until $\tilde{P}_{0}=\left(\tilde{x}_{0}, \tilde{y}_{0}\right)$ becomes a 1-median.

If in problem $\left(\mathrm{P}_{2}\right)$ the bounds $u_{i}, i=1, \ldots, n$ are chosen large enough, then problem $\left(\mathrm{P}_{2}\right)$ becomes equivalent with problem $(\mathrm{P})$ with $d=2$. Thus problem $\left(\mathrm{P}_{2}\right)$ is also $\mathcal{N} \mathcal{P}$-hard. Hence, we get the following theorem:

Theorem 5.4 Problem $\left(\mathrm{P}_{2}\right)$ is $\mathcal{N} \mathcal{P}$-hard. 
In the following we are going to solve problem $\left(\mathrm{P}_{2}\right)$ by a dynamic programming algorithm. We distinguish two cases, either only one of the four optimality conditions of Corollary 5.2 is not fulfilled, say

$$
W_{<}(X)-W_{\geq}(X)>0
$$

or two conditions are not fulfilled, say

$$
W_{<}(X)-W_{\geq}(X)>0 \quad, \quad W_{<}(Y)-W_{\geq}(Y)>0
$$

Define

$$
L(X)=\left\{i: i \in I_{<}(X), u_{i} \geq \tilde{x}_{0}-\tilde{x}_{i}\right\} \quad, \quad L(Y)=\left\{i: i \in I_{<}(Y), u_{i} \geq \tilde{y}_{0}-\tilde{y}_{i}\right\} .
$$

Let us assume that the first case holds. In this case some points $\tilde{P}_{i}=\left(\tilde{x}_{i}, \tilde{y}_{i}\right)$, $i=1, \ldots, n$, with $\tilde{x}_{i}<\tilde{x}_{0}$ are moved to $\tilde{P}_{i}^{*}=\left(\tilde{x}_{0}, \tilde{y}_{i}\right)$ by increasing their coordinates at minimum cost subject to modification bounds until $\tilde{P}_{0}$ becomes a 1 -median. Thus the first case leads to problem $\left(\mathrm{KP}_{1}\right)$ of Section 3 and can be treated in exactly the same way as outlined there. Let $s_{i}^{*}, i \in L(X)$, be an optimal solution of problem $\left(\mathrm{KP}_{1}\right) . s_{i}^{*}=1$ means that point $\tilde{x}_{i}$ is moved into $\tilde{x_{0}}$. Therefore the solution of problem $\left(\mathrm{P}_{2}\right)$ is given as:

$$
\tilde{P}_{i}^{*}= \begin{cases}\left(\tilde{x}_{i}+\left(\tilde{x}_{0}-\tilde{x}_{i}\right) s_{i}^{*}, \tilde{y}_{i}\right) & \text { if } i \in L(X), \\ \left(\tilde{x}_{i}, \tilde{y}_{i}\right) & \text { if } i \notin L(X) .\end{cases}
$$

Now we consider the second case. In this case some points $\tilde{P}_{i}=\left(\tilde{x}_{i}, \tilde{y}_{i}\right), i=$ $1, \ldots, n$, are either moved to one of the points $\tilde{P}_{i}^{*}=\left(\tilde{x}_{i}, \tilde{y}_{0}\right)$ or $\tilde{P}_{i}^{*}=\left(\tilde{x}_{0}, \tilde{y}_{i}\right)$ or to $\tilde{P}_{i}^{*}=$ $\left(\tilde{x}_{0}, \tilde{y}_{0}\right)$ by increasing their coordinates at minimum cost subject to modification bounds until $\tilde{P}_{0}=\left(\tilde{x}_{0}, \tilde{y}_{0}\right)$ becomes a 1-median. Assume that problem $\left(\mathrm{P}_{2}\right)$ has a solution. In this case it can be formulated as follows.

$$
\begin{array}{ll}
\left(\mathrm{P}_{3}\right) \quad \operatorname{minimize} & \sum_{i \in L(X)} c_{i}\left(\tilde{x}_{0}-\tilde{x}_{i}\right) s_{i}+\sum_{i \in L(Y)} c_{i}\left(\tilde{y}_{0}-\tilde{y}_{i}\right) s_{i}^{\prime} \\
\text { subject to } & \sum_{i \in L(X)} w_{i} s_{i} \geq \frac{1}{2}\left(W_{<}(X)-W_{\geq}(X)\right), \\
& \sum_{i \in L(Y)} w_{i} s_{i}^{\prime} \geq \frac{1}{2}\left(W_{<}(Y)-W_{\geq}(Y)\right), \\
& s_{i}+s_{i}^{\prime} \leq 1 \quad \text { for all } i \in T, \\
& s_{i} \in\{0,1\} \quad \text { for all } i \in L(X), \\
& s_{i}^{\prime} \in\{0,1\} \quad \text { for all } i \in L(Y),
\end{array}
$$

where $T$ is a subset of the set $L(X) \cap L(Y)$ such that its elements satisfy the following inequalities

$$
d_{1}\left(\tilde{x}_{i}, \tilde{x}_{0}\right) \leq u_{i} \quad, \quad d_{1}\left(\tilde{y}_{i}, \tilde{y}_{0}\right) \leq u_{i} \quad, \quad d_{1}\left(\tilde{P}_{i}, \tilde{P}_{0}\right) \not \leq u_{i} .
$$


Let $\left(s^{*}, s^{*^{\prime}}\right)=\left\{s_{i}^{*}, i \in L(X), s_{i}^{*^{\prime}}, i \in L(Y)\right\}$ be an optimal solution of problem $\left(\mathrm{P}_{3}\right) . s_{i}^{*}=1$ means that point $\tilde{x}_{i}$ is moved to $\tilde{x}_{0}$. Similarly, $s_{i}^{*^{\prime}}=1$ means that point $\tilde{y}_{i}$ is moved to $\tilde{y}_{0}$.

In the following we are going to present a dynamic programming algorithm for problem $\left(\mathrm{P}_{3}\right)$ which solves the problem in $O\left(n W^{2}\right)$ time. Consider problem $\left(\mathrm{P}_{3}\right)$ and assume that

$$
\alpha=\left\lceil\frac{1}{2}\left(W_{<}(X)-W_{\geq}(X)\right)\right\rceil \quad, \quad \beta=\left\lceil\frac{1}{2}\left(W_{<}(Y)-W_{\geq}(Y)\right)\right\rceil,
$$

and

$$
d_{i}=c_{i}\left(\tilde{x_{0}}-\tilde{x_{i}}\right) \text { for all } i \in L(X) \quad, \quad e_{i}=c_{i}\left(\tilde{y_{0}}-\tilde{y}_{i}\right) \text { for all } i \in L(Y) .
$$

Without loss of generality we assume that $w_{i}, i=0,1, \ldots, n$, are integers and $L(X) \cup$ $L(Y)=\{1, \ldots, n\}$. Let $f_{k}(a, b)$ be the minimum objective value of the following problem for $k=1, \ldots, n, a=0,1, \ldots, \alpha$ and $b=0,1, \ldots, \beta$ :

$$
\begin{array}{ll}
\left(\mathrm{P}_{4}\right) \text { minimize } & \sum_{i \in L(X), i \leq k} d_{i} s_{i}+\sum_{i \in L(Y), i \leq k} e_{i} s_{i}^{\prime} \\
\text { subject to } & \sum_{i \in L(X), i \leq k} w_{i} s_{i} \geq a, \\
& \sum_{i \in L(Y), i \leq k} w_{i} s_{i}^{\prime} \geq b, \\
& s_{i}+s_{i}^{\prime} \leq 1 \quad \text { for all } i \in T, i \leq k, \\
& s_{i} \in\{0,1\} \quad \text { for all } i \in L(X), i \leq k, \\
& s_{i}^{\prime} \in\{0,1\} \quad \text { for all } i \in L(Y), i \leq k .
\end{array}
$$

Thus $f_{n}(\alpha, \beta)$ is minimum objective value of problem $\left(\mathrm{P}_{3}\right)$.

We will proceed recursively to calculate $f_{n}(\alpha, \beta)$ from $f_{n-1}(\alpha, \beta)$, which in turn is calculated from $f_{n-2}(\alpha, \beta)$ and so on. The recursion is initialized with

- If $1 \in T$ and $a, b \geq 1$, then set $f_{1}(0,0)=0, f_{1}(a, b)=+\infty$ and

$$
\begin{aligned}
& f_{1}(a, 0)= \begin{cases}d_{1} & \text { if } w_{1} \geq a \\
+\infty & \text { otherwise }\end{cases} \\
& f_{1}(0, b)= \begin{cases}e_{1} & \text { if } w_{1} \geq b \\
+\infty & \text { otherwise }\end{cases}
\end{aligned}
$$


- If $1 \notin T, 1 \in L(X) \cap L(Y)$ and $a, b \geq 1$, then set $f_{1}(0,0)=0$ and

$$
\begin{aligned}
f_{1}(a, 0) & = \begin{cases}d_{1} & \text { if } w_{1} \geq a \\
+\infty & \text { otherwise }\end{cases} \\
f_{1}(0, b) & = \begin{cases}e_{1} & \text { if } w_{1} \geq b, \\
+\infty & \text { otherwise }\end{cases} \\
f_{1}(a, b) & = \begin{cases}d_{1}+e_{1} & \text { if } w_{1} \geq a, w_{1} \geq b, \\
+\infty & \text { otherwise }\end{cases}
\end{aligned}
$$

- If $1 \in L(X) \backslash L(Y)$ and $a, b \geq 1$, then set $f_{1}(0,0)=0$ and

$$
\begin{aligned}
& f_{1}(a, b)=f_{1}(0, b)=+\infty \\
& f_{1}(a, 0)= \begin{cases}d_{1} & \text { if } w_{1} \geq a \\
+\infty & \text { otherwise }\end{cases}
\end{aligned}
$$

- If $1 \in L(Y) \backslash L(X)$ and $a, b \geq 1$, then set $f_{1}(0,0)=0$ and

$$
\begin{aligned}
& f_{1}(a, b)=f_{1}(a, 0)=+\infty, \\
& f_{1}(0, b)= \begin{cases}e_{1} & \text { if } w_{1} \geq b, \\
+\infty & \text { otherwise }\end{cases}
\end{aligned}
$$

We also define

$$
\begin{aligned}
& f_{1}(a, b)=f_{1}(0,0) \quad \text { for all } a, b<0, \\
& f_{1}(a, b)=f_{1}(0, b) \quad \text { for all } a<0, b \geq 0
\end{aligned}
$$

and

$$
f_{1}(a, b)=f_{1}(a, 0) \text { for } a \geq 0, b<0 .
$$

Let us assume that $k \in T$. Now observe that if $s_{k}=0, s_{k}^{\prime}=0$ in an optimal solution to $\left(\mathrm{P}_{4}\right)$, then $f_{k}(a, b)=f_{k-1}(a, b)$. On the other hand if $s_{k}=1, s_{k}^{\prime}=0$ in an optimal solution to $\left(\mathrm{P}_{4}\right)$, then $f_{k}(a, b)=f_{k-1}\left(a-w_{k}, b\right)+d_{k}$ and if $s_{k}=0, s_{k}^{\prime}=1$, then $f_{k}(a, b)=f_{k-1}\left(a, b-w_{k}\right)+e_{k}$. Hence for $k>1, k \in T$ and $a=0,1, \ldots, \alpha$, $b=0,1, \ldots, \beta$, we obtain

$$
f_{k}(a, b)=\min \left\{f_{k-1}(a, b), f_{k-1}\left(a-w_{k}, b\right)+d_{k}, f_{k-1}\left(a, b-w_{k}\right)+e_{k}\right\} .
$$

For the other cases we can obtain $f_{k}(a, b)$ similarly. Therefore, $f_{k}(a, b)$ for $k=$ $2, \ldots, n, a=0,1, \ldots, \alpha$ and $b=0,1, \ldots, \beta$ can be calculated by

$$
f_{k}(a, b)=\left\{\begin{array}{cc}
\min \left\{f_{k-1}(a, b),\right. & \left.f_{k-1}\left(a-w_{k}, b\right)+d_{k}, f_{k-1}\left(a, b-w_{k}\right)+e_{k}\right\} \quad \text { if } k \in T, \\
\min \left\{f_{k-1}(a, b), f_{k-1}\left(a-w_{k}, b\right)+d_{k},\right. & f_{k-1}\left(a, b-w_{k}\right)+e_{k}, \\
\left.f_{k-1}\left(a-w_{k}, b-w_{k}\right)+d_{k}+e_{k}\right\} & \text { if } k \notin T, k \in L(X) \cap L(Y), \\
\min \left\{f_{k-1}(a, b), f_{k-1}\left(a-w_{k}, b\right)+d_{k}\right\} & \text { if } k \in L(X) \backslash L(Y), \\
\min \left\{f_{k-1}(a, b), f_{k-1}\left(a, b-w_{k}\right)+e_{k}\right\} & \text { if } k \in L(Y) \backslash L(X) .
\end{array}\right.
$$


For fixed $k, a$ and $b$ a constant number of calculations is needed to solve (9), hence $O(n \alpha \beta)$ calculations are required to determine $f_{n}(\alpha, \beta)$.

Given $f_{k}$ for $k=1, \ldots, n$, a recursion in the opposite direction is used to determine an optimal solution of problem $\left(\mathrm{P}_{3}\right)$. The amount of work required in the backward recursion to determine an optimal solution is dominated by the work in the forward recursion. Hence the overall running time of the algorithm is $O(n \alpha \beta)$. On the other hand, since $\alpha$ and $\beta$ are less than $W$, the running time of the algorithm is $O\left(n W^{2}\right)$. Thus we have obtained a pseudo-polynomial time algorithm for the problem $\left(\mathrm{P}_{3}\right)$.

Let $L(X) \cup L(Y) \subset\{1, \ldots, n\}$ and $|L(X) \cup L(Y)|=n^{\prime}, n^{\prime}<n$. In this case assume that $\varphi$ is a permutation of the set $L(X) \cup L(Y)$ such that $\varphi(1)<\varphi(2)<\ldots<\varphi\left(n^{\prime}\right)$. Now replace the indices $k-1$ and $k$ in the dynamic programming algorithm by $\varphi(k-1)$ and $\varphi(k)$, respectively, and compute $f_{\varphi(k)}(a, b)$ for $k=1, \ldots, n^{\prime}, a=0,1, \ldots, \alpha$ and $b=0,1, \ldots, \beta$. Note that in this case $f_{\varphi\left(n^{\prime}\right)}(a, b)$ is minimum objective value of problem $\left(\mathrm{P}_{3}\right)$.

Therefore, by considering all the concepts mentioned in this section, we conclude that problem $\left(\mathrm{P}_{2}\right)$ is $\mathcal{N} \mathcal{P}$-hard, but it can be solved by a pseudo-polynomial algorithm in $O\left(n W^{2}\right)$ time. Moreover, we conclude

Theorem 5.5 The inverse 1-median problem with variable coordinates with $(n+1)$ points in the plane endowed with the Chebyshev norm is $\mathcal{N P}$-hard, but it can be solved by a pseudo-polynomial algorithm in $O\left(n W^{2}\right)$ time, where $W$ is sum of all weights.

\subsection{The unit weight case}

Let us assume unit weights in problem $\left(\mathrm{P}_{1}\right)$, i.e., $w_{i}=1, i=0,1, \ldots, n$. In the case that the bounds $u_{i}, i=1,2, \ldots, n$, are chosen large enough, problem $\left(\mathrm{P}_{2}\right)$ can be solved in $O(n)$ time according to the solution procedure in Subsection 3.2. Otherwise the dynamic programming algorithm outlined above solves problem $\left(\mathrm{P}_{2}\right)$ in $O\left(n^{3}\right)$ time.

Proposition 5.6 The inverse 1-median problem with variable coordinates with $(n+$ 1) points in the plane under the Chebyshev norm and unit weights can be solved in $O\left(n^{3}\right)$ time.

\subsection{The unbounded case}

Dropping the last $n$ constraints of problem $\left(\mathrm{P}_{1}\right)$ and using Lemma 5.1 we have the following unbounded inverse 1-median problem with respect to the rectilinear norm in the plane.

$\left(\mathrm{P}_{5}\right) \quad$ minimize $\sum_{i=1}^{n} c_{i} d_{1}\left(\tilde{P}_{i}^{*}, \tilde{P}_{i}\right)$

subject to $\sum_{i=1}^{n} w_{i} d_{1}\left(\tilde{P}_{i}^{*}, \tilde{P}_{0}\right) \leq \sum_{i=1}^{n} w_{i} d_{1}\left(\tilde{P}_{i}^{*}, \tilde{P}\right)+w_{0} d_{1}\left(\tilde{P}_{0}, \tilde{P}\right) \quad$ for all $\tilde{P} \in \mathbb{R}^{2}$. 
Since in this problem there is not any bound constraint this problem always has a solution and the solution can be obtained through the solutions of two inverse 1-median problems on a line. Therefore, according to Section 3 we conclude that problem $\left(\mathrm{P}_{5}\right)$ is $\mathcal{N} \mathcal{P}$-hard, but it can be solved by a pseudo-polynomial algorithm in $O(n W)$ time.

Proposition 5.7 The unbounded inverse 1-median problem with variable coordinates with $(n+1)$ points in the plane under the Chebyshev norm is $\mathcal{N} \mathcal{P}$-hard, but it can be solved by a pseudo-polynomial algorithm in $O(n W)$ time, where $W$ is the sum of weights. If we assume that all the weights are equal then it can be solved in linear time.

\section{Conclusions}

We have shown that the inverse 1-median problem with variable coordinates in $\mathbb{R}^{d}$ endowed with the rectilinear norm is $\mathcal{N} \mathcal{P}$-hard, but it can be solved by a pseudopolynomial algorithm. It is also shown that the problem is polynomially solvable when the point weights are assumed to be equal. Also an efficient algorithm of running time $O(d n)$ is suggested that solves the inverse 1-median problem with variable coordinates in $\mathbb{R}^{d}$ endowed with the squared Euclidean norm. Moreover, it is shown that the inverse 1-median problem with variable coordinates in the plane endowed with the Chebyshev norm is $\mathcal{N} \mathcal{P}$-hard, but it can be solved in $O\left(n W^{2}\right)$ time.

Acknowledgment The authors thank Elisabeth Gassner and Ulrich Pferschy for their constructive suggestions.

\section{References}

[1] Alizadeh, B., R.E. Burkard, The inverse 1-center location problem on a tree, Technical Report 2009-03, Graz University of Technology, 2009.

[2] Alizadeh, B., R.E. Burkard, U. Pferschy, Inverse 1-center location problems with edge length augmentation on trees, Technical Report 2009-07, Graz University of Technology, 2009.

[3] Burkard, R.E., M. Galavii, E. Gassner, The inverse Fermat-Weber problem. Technical Report 2008-14, Graz University of Technology, 2008.

[4] Burkard, R.E., C. Pleschiutschnig, J. Zhang, Inverse median problems. Discrete Optimization 1, 2004, 23-39.

[5] Burkard, R.E., C. Pleschiutschnig, J.Z. Zhang, The inverse 1- median problem on a cycle. Discrete Optimization 5, 2008, 242-253.

[6] Balas, E., E. Zemel, An algorithm for large zero-one knapsack problem. Operations Research 28, 1980, 1130-1154.

[7] Burton, D., Ph.L. Toint, On an instance of the inverse shortest path problem. Mathematical Programming 53, 1992, 45-61. 
[8] Cai, M.C., X.G. Yang, J.Z. Zhang, The complexity analysis of the inverse center location problem. Journal of Global Optimization 15, 1999, 213-218.

[9] Gassner, E., The inverse 1-maxian problem with edge length modification. Journal of Combinatorial Optimization 16, 2008, 50-67.

[10] Hamacher, H.W, Mathematische Lösungsverfahren für planare Standortprobleme, Braunschweig/Wiesbaden: Vieweg, 1995.

[11] Heuberger, C., Inverse optimization: a survey on problems, methods, and results. J. Combinatorial Optimization 8, 2004, 329-361.

[12] Kellerer, H., U. Pferschy, D. Pisinger, Knapsack problems, Berlin: Springer, 2004.

[13] Martello, S., P. Toth, Knapsack problems: algorithms and computer implementations, Chichester: John Wiley, 1990.

[14] Yang, X., J. Zhang, Inverse center location problem on a tree. Journal of Systems Science and Complexity 21, 2008, 651-664. 